\title{
Role of Magnetic Anchors During Laparoendoscopic Single Site Surgery and NOTES
}

\author{
Jay D. Raman, M.D., ${ }^{1,2}$ Daniel J. Scott, M.D., ${ }^{3}$ and Jeffrey A. Cadeddu, M.D. ${ }^{2}$
}

\begin{abstract}
Evolution of minimally invasive techniques has generated an impetus in the surgical community to reduce the invasiveness of laparoscopic surgery. To achieve this goal, surgeons are either limiting the number of transabdominal trocars using laparoendoscopic single site surgery (LESS) or eliminating them completely using natural orifice translumenal endoscopic surgery (NOTES). The principle challenge with LESS or NOTES procedures is loss of triangulation, reduction in instrument working envelopes, and collision of conventional laparoscopic instrumentation. To overcome these limitations, surgical innovations, such as the use of deployable intracorporeal instrumentations, are necessary. Magnetic anchoring and guidance system (MAGS) technology is one approach for deployable instrumentation whereby intra-abdominal instruments can be maneuvered by the use of an external handheld magnet. Such a strategy would permit a single access port (either transabdominal or via a natural orifice) to serve as an entry point for multiple instruments. MAGS technology has thus far developed to incorporate instruments such as retractors, an intra-abdominal camera, and cautery dissectors. Continuous instrument development and rapid prototyping have created more robust MAGS platforms while potentially obviating the need for laparoscopic assistance. Animal work to date is promising with successful completion of a single-port nephrectomy as well as a NOTES transvaginal cholecystectomy in both nonsurvival and survival porcine models. As with all new technology, there will be an associated learning curve for these MAGS techniques that will necessitate more thorough elucidation before widespread use. The development of magnetically controlled and anchored intracorporeal surgical instruments, retractors, and cameras may advance surgical practice and improve patient care while meeting or exceeding the benefits of current-day fixed-trocar laparoscopy.
\end{abstract}

\section{Evolution of Laparoscopy}

$\mathbf{W}$ HILE OPEN APPROACHES have historically been the gold standard for the surgical management of benign and malignant diseases, considerable morbidity and delayed convalescence occurs after such procedures. The introduction of laparoscopic techniques in the late 1980s and early 1990s has ushered in a novel era in the surgical management of human diseases. ${ }^{1}$

Advantages of laparoscopic surgery in comparison with open surgery are well established. ${ }^{2,3}$ Conversion from an open to a closed operation has significantly decreased pain, morbidity, hospital duration, and cost of many commonly performed procedures. Indeed, minimally invasive approaches have increasingly become the new standard of care for many procedures across all surgical disciplines (cholecystectomy, nephrectomy, oopherectomy, etc).
Triangulation is one of the fundamental concepts of conventional laparoscopic surgery. Adequate external placement of trocars is necessary for intracorporeal spacing of instrumentation to facilitate traction and anatomic dissection. Further, triangulation minimizes collision or "sword fighting" of laparoscopic instruments both externally and internally. Conventional laparoscopy is also limited by the fixed working envelope around each port secondary to the fulcrum motion around the insertion point of each trocar.

Current robotic systems can overcome some of these hurdles, but they still require multiple trocars and are frequently restricted by even smaller working envelopes. Thus, while laparoscopy undoubtedly decreases surgical morbidity, it still requires three to four spaced incisions each at least 1 to $2 \mathrm{~cm}$ in length. Further, each working port carries morbidity risks of bleeding, hernia, and/or internal organ damage, and incrementally decreases cosmesis. ${ }^{4,5}$ Cosmesis is particularly

\footnotetext{
${ }^{1}$ Division of Urology, Penn State, Milton S. Hershey Medical Center, Hershey, Pennsylvania.

Departments of ${ }^{2}$ Urology and ${ }^{3}$ Surgery, University of Texas - Southwestern Medical Center, Dallas, Texas.
} 
important in procedures performed on pediatric patents and is also demanded by sophisticated adult patients. ${ }^{6}$

\section{Recent Advances}

Recently, there has been an impetus in the surgical community to reduce the invasiveness of laparoscopic surgery. To achieve this goal, surgeons are either decreasing the number of trocars placed through the abdominal wall or eliminating them completely.

Natural orifice translumenal endoscopic surgery (NOTES) has been described as the next surgical frontier with the objective of incision-free abdominal surgery. NOTES approaches abdominal surgery through natural orifices (mouth, vagina, and rectum), thus obviating external abdominal scars. Animal models have been used to demonstrate the potential applications of NOTES, including transgastric and transvesical peritoneoscopy, transvaginal tubal ligation, hysterectomy, and cholecystectomy. ${ }^{7,8}$ Recently, two transvaginal cholecystectomies in humans have been described. The first occurred in New York, NY, in a 66-year-old patient, ${ }^{9}$ and the second in Strasbourg, France, in a 30-year-old patient. ${ }^{10}$ Subsequent preliminary clinical series in human patients with transvaginal NOTES cholecystectomy similarly appear promising. ${ }^{11}$

The first urologic application of NOTES was by Gettman and colleagues ${ }^{12}$ in 2002; these investigators reported the successful completion of six laparoscopic transvaginal nephrectomies using conventional instrumentation in a porcine model. Five nephrectomies, however, still needed use of one conventional laparoscopic port. Further, they noted that limitations of standard laparoscopic and endourologic instrumentation made the procedure "cumbersome and time consuming."

A key requirement of NOTES surgery, as identified by the Natural Orifice Surgery Consortium for Advancement and Research, is a stable surgical platform to support and guide flexible endoscopes and instruments. Modern instrumentation has proved to be limited in providing a suitable platform for advanced NOTES surgery, which has resulted in this technique languishing for several years.

More recently, Clayman and associates ${ }^{13}$ reported their experience with single-port NOTES nephrectomy using a purpose-built multilumen transvaginal operating platform (TransPort ${ }^{\mathrm{TM}}$ Multi-Lumen Operating Platform, USGI Medical, San Clemente, CA). This flexible device has four working channels and can be locked into position, thereby creating a rigid multitasking platform that allows two-handed tissue manipulation. While appearing promising, potential drawbacks to NOTES surgery are not insignificant. Operative duration is significantly longer than conventional laparoscopy, specialized equipment is necessary, and there is a steep learning curve for newer instrumentation and operative approach.

An alternative to conventional laparoscopy and NOTES is laparoendoscopic single site surgery (LESS), which uses articulating or bent instrumentation with specialized multilumen ports. Such instrumentation would obviate the need to space trocars for triangulation, allowing for the creation of a small, solitary portal of entry into the abdomen. LESS has been reported in the literature for appendectomy ${ }^{14}$ and cholecystectomy ${ }^{15}$ since 1998, although the approach failed to gain momentum for years because of technical limitations with conventional instrumentation.

Technical innovations described above have ushered in a recent renaissance for LESS. Raman and coworkers ${ }^{16}$ recently reported their initial experience with LESS nephrectomy in a porcine model and in human subjects. Other small clinical series have further supported the feasibility, safety, and successful completion of LESS for urologic procedures, including nephrectomy, adrenalectomy, pyeloplasty, renal cryotherapy, and sacrocolpopexy. ${ }^{17}$

Although the early experiences for NOTES and LESS are promising, an experienced laparoscopic skill set is essential for the safe and effective completion of the procedures. More significantly, adequate instrumentation continues to be a surgical challenge. While articulating/bent instrumentation and multichannel ports are a promising innovation, early surgical experience suggests that instrument collision, both internally and externally, is a constant challenge. ${ }^{16,17}$

\section{Deployable Intracorporeal Instruments}

The principle challenge with LESS or NOTES procedures is loss of triangulation and reduction in instrument working envelopes. To overcome these problems, new instrumentation must be developed and evaluated before single trocar or NOTES procedures will be readily used. One such innovation involves use of numerous small surgical instruments that can be deployed intracorporeally. Such a strategy would permit a single access port (either transabdominal or via a natural orifice) to serve as an entry point for multiple instruments.

The most obvious target for such deployable instrumentation involves video imaging systems. A deployable camera would obviate the need for a traditional laparoscope, thus permitting visualization of the operative field while the access port was used for other instrumentation. Researchers from Columbia University (New York, NY) have designed a novel stereoscopic three-dimensional (3D) imaging device that can be inserted and attached in the body cavity using a peritoneal clip. ${ }^{18}$ This novel robotic camera is totally insertable and includes two motorized, remotely controlled cameras with 5 degrees of freedom each. The cameras collapse within the shell for insertion and are exposed once inside the abdomen. Moved in concert, the cameras provide 3D imaging; alternatively, they can be moved separately, providing simultaneous two-dimensional views of two different areas. Preliminary results using a validated laparoscopic assessment tool (McGill Inanimate System for Training and Evaluation of Laparoscopic Skills) demonstrated that the new robotic camera prototype allowed for equivalent performance when compared with a conventional laparoscope. ${ }^{19}$

Similar work from the University of Nebraska has resulted in the development of a deployable video imaging platform termed in-vivo camera robots. ${ }^{20}$ The mobile system (Mobile Adjustable-focus Robotic Camera or MARC) can be deployed through an access port and maneuvered throughout the abdominal cavity to provide visualization. Incorporated into the housing of the device are two helical wheels that allow the robot to propel itself over abdominal organs with capabilities of going forward, reverse, or turning. Thus, the MARC instrument is capable of "roaming" around the abdomen while providing real-time video imaging from any location. 
In experimental models, injuries to abdominal organs from the device's wheels have not been noted. One notable limitation, however, is that the camera view from the MARC instrument is either directed toward tissue very close to the camera in a downward direction or toward nonadjacent tissue in an upward viewing fashion. As such, the traditional panoramic view readily used during most laparoscopic procedures is not possible.

\section{Magnetic Anchoring for Intracorporeal Instruments}

\section{Background}

At the University of Texas (UT Southwestern Medical Center and UT Arlington), we have pursued an alternative approach for deployable instrumentation by developing a magnetic anchoring and guidance system (MAGS). MAGS instruments are designed to allow maneuvering of intraabdominal instruments by use of an external handheld magnet, whereby magnetic interaction forces allow coupling of the internal and external components across the abdominal wall without the need for incisions or trocars (Fig. 1).

Magnetic anchoring technology involves the use of an internal component consisting of a surgical instrument attached to a baseplate (containing permanent magnets) and an external component consisting of a handheld permanent magnet. The two components are held together via magnetic coupling such that the internal component may be directly manipulated by moving the external magnet. Early work involved the development of a platform with permanent magnets made from neodymium-iron-boron, which were focused and shielded to optimize strength and generate sufficient coupling forces to control 25 to 45 gm instruments. ${ }^{21}$ Once the internal component is positioned in a desired anatomic location, the external component can be optionally exchanged for an 18-gauge percutaneous, threaded needle anchor to provide secure, hands-free fixation.

\section{Instrumentation}

MAGS technology has thus far developed to incorporate instruments such as retractors, an intra-abdominal camera, and cautery dissectors.

Several types of passive tissue retractors were designed for successful introduction through a $15-\mathrm{mm}$ diameter standard trocar. $^{22-24}$ To elevate and move organs, such as the liver or spleen, a sling retractor consisting of latex tubing of various lengths was attached to two internal MAGS platforms. By manipulating the pair of external anchors, the surgeon can position the sling under an organ to elevate it. To elevate or retract laterally, a prototype paddle-type retractor attached to a pair of internal MAGS platforms that contain two coupling magnets was designed (Fig. 2). Manipulation of the external magnet permits positioning of the retractor as well as the ability to actively depress and/or elevate the internal retractor. For general surgical applications, gallbladder retraction can occur via an attachable magnet and a flexible grasper cradle.

A MAGS camera was designed by packaging a commercially available miniature camera (QN42H, Elmo, Plainview, NY) within a customized aluminum enclosure attached to a pair of internal MAGS platforms ${ }^{23,24}$ (Fig. 3). The camera can be inserted through a conventional 15-mm trocar and coupled to the external magnets. Manipulating the pair of external magnets in conjunction enables horizontal camera orientation (yaw), while pushing the external magnets apart or together allows for the camera lens to be actively deflected upward or downward (pitch).

Because of its intended nonmedical design, MAGS camera resolution has thus far been inferior to that of conventional laparoscopes. While movement of the anchors provides the desired yaw and pitch control, the camera has a fixed focus, making positioning critical. Further, lens fogging and light shadowing secondary to delivery of light not parallel to the camera are current challenges with this technology.

MAGS cautery dissectors have been designed in several different configurations. ${ }^{22}$ Early experience noted springloaded designs were prone to lodging on surrounding tissues during insertion and removal. As such, engineering modifications were made that permitted instruments to be deployed in a collapsed configuration with extension of the cautery arm into a 45-degree angle when coupled to the abdominal wall.

Initial designs used a mechanism whereby the cautery arm was held in the closed position using a magnet and biopsy forceps controlled via the endoscope were used for intracorporeal arm deployment. A subsequent design used a pneumatic piston to activate the arm via compressed air. Most recently, a pneumatically powered robotic arm remotely controlled using a joystick (with 8 degrees of freedom; 3 on board and 5 via magnetic manipulation) was designed to support a conventional hook cautery attachment ${ }^{24}$ (Fig. 4). This robotic arm can be deployed through a 15-mm trocar and then anchored to the abdominal wall by an externally placed

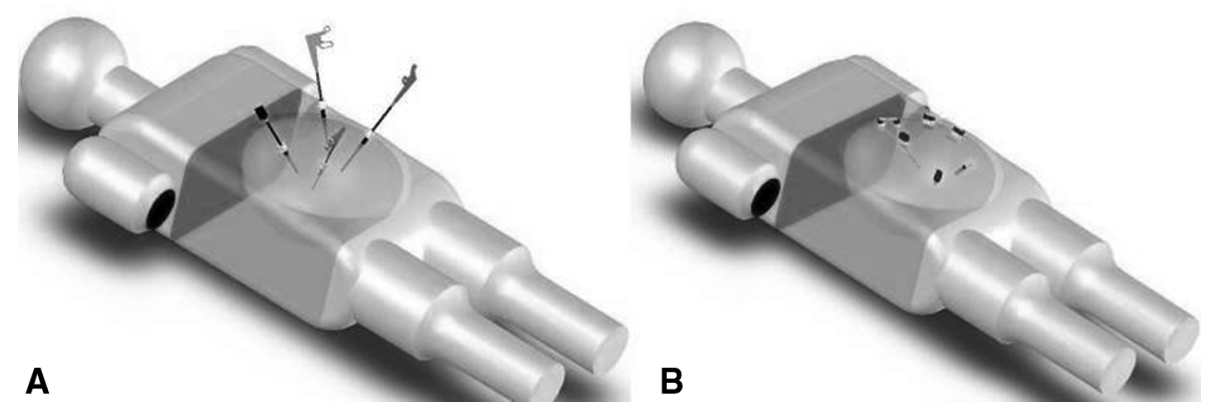

FIG. 1. Schematic representation (A) of conventional laparoscopic surgery (left) and (B) laparoendoscopic single site surgery (LESS) through which multiple magnetic anchoring and guidance system (MAGS) instruments are introduced and deployed (right). 
A
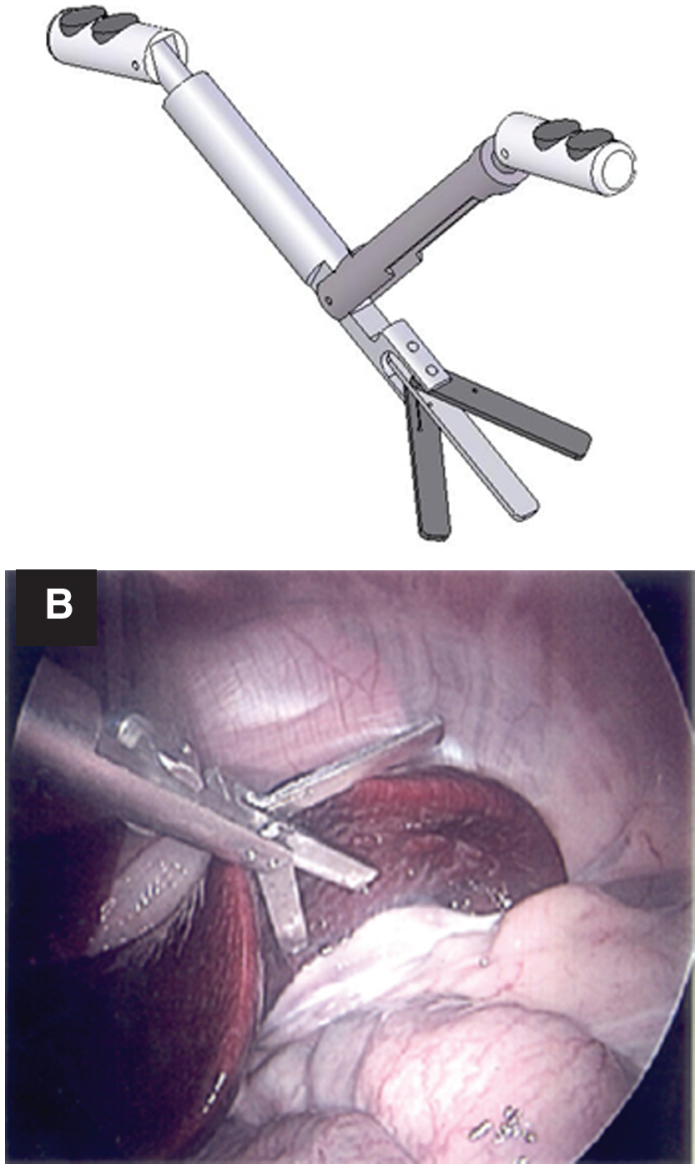

FIG. 2. Schematic (A) and intra-abdominally (B) deployed view of intracorporeal MAGS retractor as shown elevating a porcine spleen.

magnet. All cauterizers were maneuvered using an external magnet, and deformation of the abdominal wall allowed angulations during dissection.

\section{Laboratory experience}

Before in-vivo experimentation, MAGS platform coupling strength was determined across various distances of air gaps, across ex-vivo porcine abdominal walls, and commercially purchased bovine muscle of various thicknesses. After establishment of proof-of-concept, numerous animal experiments have allowed for the successful pioneering of nonsurvival and survival surgery using MAGS instrumentation.

Early work using MAGS camera and retractor instruments facilitated completion of a minimally invasive nephrectomy in a nonsurvival porcine model while reducing the number of laparoscopic trocars to two. ${ }^{23}$ With the addition of a MAGS robotic cautery dissector, the number of laparoscopic trocars was reduced to a single port in a subsequent study. ${ }^{24}$ For the laparoscopic approach, electrical (camera and cauterizer) and pneumatic (cauterizer) tethers are fed externally for signal transmission, electrical energy, and robotic control.

Subsequent work has furthered research using the MAGS tool-suite for general surgical and NOTES applications. ${ }^{22,25,26}$ Several generations of MAGS cameras, tissue retractors, and cauterizer instruments have been successfully deployed via
A
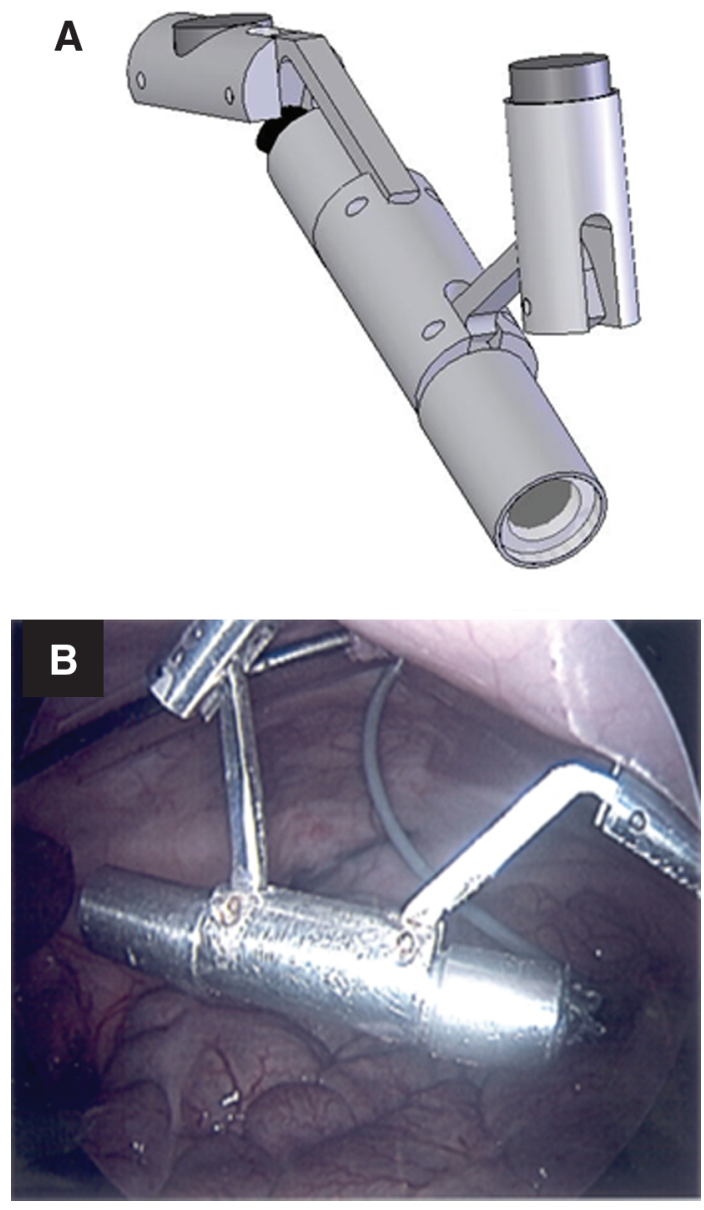

FIG. 3. Schematic (A) and intra-abdominally (B) deployed view of intracorporeal MAGS camera.

transabdominal and NOTES (transgastric, transcolonic, and transvaginal) approaches for use in a porcine cholecystectomy model. In all cases, the gallbladder has been successfully dissected free from the liver attachments using only the MAGS cauterizer.

Continuous instrument development and rapid prototyping has created more robust MAGS platforms while potentially obviating the need for laparoscopic assistance. Using the MAGS retractor and cauterizer, along with a standard single-channel flexible endoscope (for visualization, suctionirrigation, clips, grasper, and snare), these investigators have successfully performed completely NOTES transvaginal cholecystectomy on both nonsurvival and survival (14-day) porcine models with no complications or infections. ${ }^{26}$

\section{Technical limitations}

Before widespread adoption of the MAGS platform, both clinical and engineering limitations must be addressed. Surgeons must become familiar with the MAGS components both in a dry laboratory and in animal models. As with all new technology, there will be an associated learning curve, and it will be incumbent on surgeons to develop new MAGS techniques by modifying traditional laparoscopic modalities.

In addition, the optimal number of external magnets in the operating area (abdominal wall) remains to be determined. Theoretically, it is limited only by the working area of the 
A

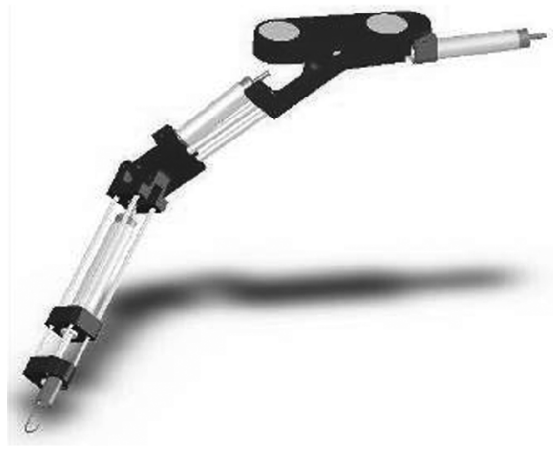

B

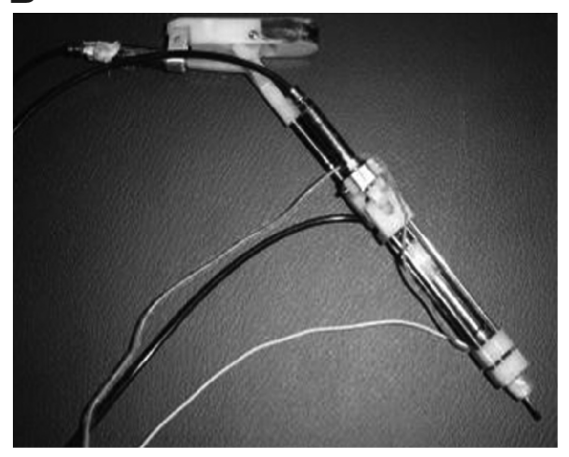

C

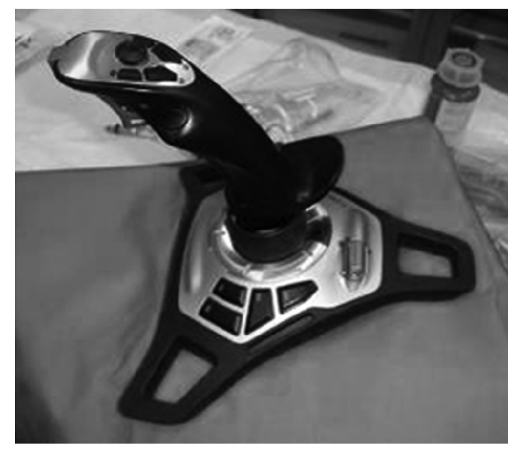

FIG. 4. Schematic (A) and ex vivo (B) view of MAGS robotic cautery dissector that can be remotely controlled via a joystick (C).

abdominal wall minus the area used by the external magnet. Closer distances, however, may generate magnet-magnet interference as well as operator hand-magnet collisions. Such interactions may result in inadvertent magnetic coupling between instruments with resultant retractor dislodgement. Clearly, these issues will have to be elucidated during the learning curve phase of MAGS development before widespread use.

The coupling strength of magnetics (electromagnetic or permanent magnets) decreases as a decaying exponential with respect to the distance between the source magnet and its target. Currently, tissue thicknesses in excess of $1.5 \mathrm{~cm}$ limit the effectiveness of the paddle retractor, while the camera can be supported up to tissue thicknesses of $2.5 \mathrm{~cm}$. As such, present clinical use of MAGS technology would be restricted to thin or pediatric patients. Future directions are needed to develop electromagnets capable of generating stronger magnetic fields.

Finally, additional work is needed to develop a more robust MAGS camera system. Current laboratory work has been limited by fogging of the camera and a lack of sufficient lighting (despite on-board light-emitting diode). Some cases have required laparoscope and flexible endoscope assistance for visualization. Purpose-built modifications in camera design are necessary to obviate the need for additional lighting sources.

\section{Future Directions}

The development of magnetically controlled and anchored intracorporeal surgical instruments, retractors, and cameras may advance surgical practice and improve patient care while meeting or exceeding the benefits of current fixed-trocar laparoscopy. The MAGS technology, however, will be a significant advance only if certain future objectives are achieved. First, instruments must retain the advantages of conventional and robotic instrumentation, while being easily repositioned when the working envelope becomes disadvantageous. Second, surgical pain should be decreased by the elimination of most and possibly all abdominal trocars. Third, mechanisms must be developed for simple deployment and retrieval of intra-abdominal robots and instruments. Finally, future MAGS platforms must be able to provide a local power source with wireless operating controls such that instruments are completely independent of the deployment site.
Ultimately, wireless and robotic technology may allow full realization of the concept of intracorporeal deployment of a wide array of highly effective surgical instruments. With miniaturized equipment and powerful on-board or transmitted energy sources, complex intracorporeal surgery may be possible without the use of abdominal incisions.

\section{Conclusions}

The evolution of NOTES and LESS procedures has created a need for technical innovations whereby surgical instruments can be deployed intracorporeally. Magnetically controlled and anchored instrumentation is a novel, promising surgical strategy for such deployable instrumentation. While early experience is promising, future work is necessary to build more robust MAGS platforms to facilitate simple deployment, positioning, and retrieval of intra-abdominal robots and instruments. Ultimately, improvement in MAGS technology may allow full realization of surgery without abdominal incisions.

\section{Disclosure Statement}

Jeffrey A. Cadeddu is the patent holder for MAGS technology.

\section{References}

1. Clayman RV, Kavoussi LR, Soper NJ, et al. Laparoscopic nephrectomy: Initial case report. J Urol 1991;146:278-282.

2. Dunn MD, Portis AJ, Shalhav AL, Elbahnasy AM, Heidorn C, McDougall EM, Clayman RV. Laparoscopic versus open radical nephrectomy: A 9-year experience. J Urol 2000;164: 1153-1159.

3. Rassweiler J, Frede T, Henkel TO, Stock C, Alken P. Nephrectomy: A comparative study between the transperitoneal and retroperitoneal laparoscopic versus the open approach. Eur Urol 1998:33:489-496.

4. Lowry PS, Moon TD, D'Alessandro A, Nakada SY. Symptomatic port-site hernia associated with a non-bladed trocar after laparoscopic live-donor nephrectomy. J Endourol 2003; 17:493-494.

5. Marcovici I. Significant abdominal wall hematoma from an umbilical port insertion. JSLS 2001;5:293-295.

6. Dunker MS, Stiggelbout AM, van Hogezand RA, Ringers J, Griffioen G, Bemelman WA. Cosmesis and body image after 
laparoscopic-assisted and open ileocolic resection for Crohn's disease. Surg Endosc 1998;12:1334-1340.

7. Wagh MS, Thompson CC. Surgery insight: Natural orifice translumenal endoscopic surgery-an analysis of work to date. Nat Clin Pract Gastroenterol Hepatol 2007;4:386392.

8. Gettman MT, Blute ML. Transvesical peritoneoscopy: Initial clinical evaluation of the bladder as a portal for natural orifice translumenal endoscopic surgery. Mayo Clin Proc 2007; 82:843-845.

9. Grady D. Doctors try new surgery for gallbladder removal. New York Times. 2007.

10. Marescaux J, Dallemagne D, Perretta S, Wattiez A, Mutter D, Coumaros D. Surgery without scars: Report of translumenal cholecystectomy in a human being. Arch Surg 2007;142:823827.

11. Zorron R, Maggioni LC, Pombo L, Oliveira AL, Carvalho GL, Filgueiras M. NOTES transvaginal cholecystectomy: Preliminary clinical application. Surg Endosc 2008;22(2):542547.

12. Gettman MT, Lotan Y, Napper CA, Cadeddu JA. Transvaginal laparoscopic nephrectomy: Development and feasibility in the porcine model. Urology 2002;59:446-450.

13. Clayman RV, Box GN, Abraham JB, et al. Rapid communication: Transvaginal single-port NOTES nephrectomy: Initial laboratory experience. J Endourol 2007;21:640-644.

14. Esposito C. One-trocar appendectomy in pediatric surgery. Surg Endosc 1998;12:177-178.

15. Piskun G, Rajpal S. Transumbilical laparoscopic cholecystectomy utilizes no incisions outside the umbilicus. J Laparoendosc Adv Surg Tech A 1999;9:361-364.

16. Raman JD, Bensalah K, Bagrodia A, Stern JM, Cadeddu JA. Laboratory and clinical development of single keyhole umbilical nephrectomy. Urology 2007;70:1039-1042.

17. Tracy CR, Raman JD, Cadeddu JA, Rane A. Laparoendoscopic single site surgery in urology: where have we been and where are we heading. Nat Clin Pract Urol 2008;10:561568.

18. Miller A, Allen P, Fowler DL. In-vivo steroscopic imaging system with 5 degrees-of-freedom for minimal access surgery. In: Westwood JD, ed, Medicine Meets Virtual Reality 12 IOS Press, 2004:234-240.

19. Strong VE, Hogle NJ, Fowler DL. Efficacy of novel robotic camera vs a standard laparoscopic camera. Surg Innov 2005; 12:315-318.
20. Rentschler ME, Dumpert J, Platt SR, Ahmed SI, Farritor SM, Oleynikov D. Mobile in vivo camera robots provide sole visual feedback for abdominal exploration and cholecystectomy. Surg Endosc 2006;20:135-138.

21. Cadeddu JA, Eberhart R, Fernandez R, Bergs R. Transabdominal magnetic anchoring system for trocar-less laparoscopic surgery. J Urol 2002;167(suppl):4.

22. Scott DJ, Tang SJ, Fernandez R, Bergs R, Goova MT, Zeltser I, Kehdy FJ, Cadeddu JA. Completely transvaginal NOTES cholecystectomy using magnetically anchored instruments. Surg Endosc 2007;21:2308-2316.

23. Park S, Bergs RA, Eberhart R, Baker L, Fernandez R, Cadeddu JA. Trocar-less instrumentation for laparoscopy: Magnetic positioning of intra-abdominal camera and retractor. Ann Surg 2007;245:379-384.

24. Zeltser IS, Bergs R, Fernandez R, Baker L, Eberhart R, Cadeddu JA. Single trocar laparoscopic nephrectomy using magnetic anchoring and guidance system in the porcine model. J Urol 2007;178:288-291.

25. Scott DJ, Tang SJ, Fernandez R, Bergs R, Cadeddu JA. Transgastric, transcolonic, and transvaginal cholecystectomyusing magnetically anchored instruments. Surg Endosc 2007;21(suppl):S474.

26. Scott DJ, Tang SJ, Goova MT, et al. Short-term survival outcomes following transvaginal NOTES cholecystectomy using magnetically anchored instruments. Gastrointest Endosc 2007;65:AB109.

$$
\begin{array}{r}
\text { Address reprint requests to: } \\
\text { Jeffrey A. Cadeddu, M.D. } \\
\text { Department of Urology } \\
\text { UT Southwestern Medical Center } \\
5323 \text { Harry Hines Blvd. } \\
\text { J8.106, MC } 9110 \\
\text { Dallas, TX 75390-9110 }
\end{array}
$$

E-mail: jeffrey.cadeddu@utsouthwestern.edu

$\begin{aligned} & \text { Abbreviations Used } \\ & \text { MAGS }=\text { magnetic anchoring and guidance system } \\ & \text { MARC }=\text { Mobile Adjustable-focus Robotic Camera } \\ & \text { NOTES }=\text { natural orifice translumenal endoscopic surgery } \\ & \text { LESS }=\text { laparoendoscopic single site surgery }\end{aligned}$

